\title{
Hydrothermal Aging Life Model of Composite Solid Propellant Based on Modified Arrhenius Method
}

\author{
Du Yongqiang ${ }^{1, a}$, Zheng Jian ${ }^{1}$, Peng Wei ${ }^{1}$, Zhang Xiao ${ }^{1}$ \\ ${ }^{1}$ Mechanical Engineering College, Shijiazhuang, 050003, Hebei, China \\ ${ }^{a}$ Corresponding author
}

\begin{abstract}
By combining with the quantum mechanics theory on the relationship between the electronic equipment aging reaction rate and temperaturehumidity, a hydrothermal aging life model based on modified Arrhenius method is established with the propellant mechanical properties as the research object. According to experimental data, the empirical formula is obtained, and the model is used to predict the storage life of a solid propellant at 25 and $50 \%$ humidity conditions, which result is 11.7 a.
\end{abstract}

\section{Introduction}

The composite solid propellant, as a high filler particle composite material, has changed the physical and chemical properties under the storage period. The mechanical properties of the propellant will deteriorate with the increase of storage time [1]. At present, the research method is to accelerate the aging process, but this method has no consideration of the effect of humidity on the aging of propellant, but only the temperature as the main factor. In the actual storage, the propellant is a kind of material with strong moisture absorption ability. At the same time, the traditional Arrhenius equation needs to be modified to meet the practical requirements because of its limitations.

Zhao Feng et al. [2] has established the hydrothermal aging life model with the propellant mechanical properties as the research object and has obtained the experiential formula by fitting experimental datum. Zhang Lei et al. [3] combined the Eying model with Arrhenis aging model and obtained the hydrothermal aging life model, and the storage life of a solid propellant under temperature $20^{\circ} \mathrm{C}$ and $50 \%$ humidity condition was predicted, which is similar to the actual storage span. Zhang Xiao-jun et al. [4] developed the hydrothermal aging life model for adhesion interface and predicted the life in normal storage condition. However, the modified Arrhenius equation is not included in the hydrothermal aging life model. In this paper, the modified Arrhenius equation is analyzed, and the relationship between the aging reaction rate and the temperature and humidity of the electronic products [5] is analyzed. The hydrothermal aging life model of the propellant was deduced, and the feasibility of the model was verified by experimental data.

\section{Modified Arrhenius equation}

\subsection{Traditional Arrhenius equation and its limitations}

In the high temperature accelerated aging test, the Arrhenius model is the most commonly used, and the model derived from the Swedish physical chemist Svante Arrhenius in 1887 [6]. The reaction rate equation is:

$$
K=A e^{-E_{a} / R T}
$$

In formula: A-the pre exponential factor; $E_{a}$-apparent activation energy; $\mathrm{R}$ - molar gas constant; $\mathrm{T}$ thermodynamic temperature.

It can be seen From the formula (1) that the pre exponential factor $\mathrm{A}$ and the apparent activation energy $E_{a}$ in the traditional Arrhenius equation is assumed as constant, which is not in conformity with the actual situation. In fact, these two parameters are closely related to temperature, especially the apparent activation energy, which is located in the position of the index, and once changing, it will affect the accuracy of the estimated results. So it is necessary to revise the Arrhenius equation.

\subsection{The modified Arrhenius Equation}

The apparent activation energy expression is obtained by taking the logarithm to formula (1):

$$
E_{a}=R T^{2} \frac{d \ln K}{d T}=-R \frac{d \ln K}{d\left(\frac{1}{T}\right)}
$$

According to empirical formula:

$$
\frac{d \ln K_{1}}{d T}-\frac{d \ln K_{-1}}{d T}=\frac{\overline{E_{P}}}{R T^{2}}-\frac{\overline{E_{R}}}{R T^{2}}=\frac{Q_{v}}{R T^{2}}
$$

In formula: $K_{1}$-positive reaction rate; $K_{-1}$-reverse reaction rate; $\overline{E_{P}}$-generation mean energy; $\overline{E_{R}}$-reactant average energy; $Q_{v}$ - heat capacity.

According to the differential equation of Arrhenius theorem [7]:

$$
\frac{d \ln K_{1}}{d T}-\frac{d \ln K_{-1}}{d T}=\frac{E_{a}}{R T^{2}}-\frac{E_{a}^{\prime}}{R T^{2}}
$$

Combined (3) and (4), it can be obtained:

$$
\overline{E_{P}}-\overline{E_{R}}=E_{a}-E_{a}^{\prime}=Q_{v}
$$

In the formula: $E_{a}$-the activation energy of the forward reaction; $E_{a}^{\prime}$-the activation energy of the reverse reaction. 
By formula (5), it can be obtained that $E_{a}=E_{a}^{\prime}+Q_{v}$. In the formula, is the function of temperature, so $E_{a}$ is also the function of temperature. Therefore, it is not practical to assume the activation energy in the traditional Arrhenius equation as constant. According to the related research, the Arrhenius equation can be modified as the three parameter formula [8]:

$$
K=A T^{m} \exp \left(-\frac{E}{R T}\right)
$$

In the formula: $\mathrm{M}, \mathrm{E}$, and $\mathrm{A}$ are all undetermined coefficients. The formula is modified Arrhenius equation. Combined formula (2) and (6), it can be obtained:

$$
E_{a}=-R \frac{m d \ln T-\frac{E}{R} d\left(\frac{1}{T}\right)}{d\left(\frac{1}{T}\right)}=E+m R T
$$

By the formula (7), it can be seen that there is a linear relationship between the activation energy $E_{a}$ and temperature $\mathrm{T}$.

\section{Deduction of hydrothermal aging life model}

Both the traditional Arrhenius equation and the modified Arrhenius equation can only be used to evaluate the situation in which the temperature is the main factor. When the failure stress is the other stress, the Eyring model is generally used:

$$
L(V)=\frac{1}{V} e^{-\left(\mathrm{A}-\frac{B}{V}\right)}
$$

In the formula, $\mathrm{V}$-the stress value of the absolute unit (e.g. relative humidity, etc.); $\mathrm{A}$ and $\mathrm{B}$ are the parameters of the model.

The formula can be transformed into:

$$
L(V)=\frac{e^{-A}}{V} e^{\frac{B}{V}}=\frac{1}{V} \text { Const } \cdot e^{\frac{B}{V}}
$$

Contrasting formula (6) and (9), it can be seen that the Eyring model and Arrhenius model are similar, and combining the above two models, hydrothermal aging life model is obtained:

$$
L(H, T)=\frac{A}{H} T^{m} e^{\frac{b}{H}+\frac{c}{T}}
$$

In the formula, $L(H, T)$-the average life span, or the median life span; m, B, C and A -the model parameters ; $\mathrm{H}$ - Relative humidity (fraction or percentage); T-absolute temperature $(\mathrm{K})$.

If the temperature $\mathrm{T}$ in the formula (10) is constant, the formula is transferred into :

$$
L\left(H, T_{0}\right)=\frac{A}{H} T_{0}^{m} e^{\frac{b}{H}+\frac{c}{T_{0}}}=\frac{A T_{0}^{m} e^{\frac{c}{T_{0}}}}{H} e^{\frac{b}{H}}
$$

Which has the similar form with the Eyring model (8). And when the humidity $\mathrm{H}$ is constant, the formula (10) can be transferred into :

$$
L(H, T)=\frac{A}{H_{0}} T^{m} e^{\frac{b}{H_{0}}+\frac{c}{T}}=\frac{A e^{\frac{b}{H_{0}}}}{H_{0}} T^{m} e^{\frac{c}{T}}
$$

Which has the similar form with the modified Arrhenius equation (6).

\section{Model parameter fitting}

Since the activation energy is related with temperature and at the same temperature, the activation energy can be assumed to be constant, so the fitting of the parameters can be separated to reduce the actual operation amount. Zhang Hao et al. [9] studied the linear activation energy method to estimate the life of the propellant, Luo Huaide et al. [10] studied the rapid prediction of the life of the propellant, and prove that the results of two points methods are close to the conventional method. Combing he Arrhenius equations of the two test temperature, then the formula can be obtained as follow :

$$
E_{a}=\frac{R T_{2} T_{1}}{T_{2}-T} \ln \frac{K_{2}}{K_{1}}
$$

If the reaction rate constant of the accelerated aging tests at two temperatures is known, it is easy to find the activation energy, and then the life prediction is carried out. In this paper, the $\mathrm{m}$ values of the parameters in the formula (10) are solved by two points method. The relevant data are derived from the literature $[2,10]$.

The activation energy obtained by the two points method is shown in the following table 1 .

Tab 1.Results of activation energy calculated by two points method

\begin{tabular}{|c|c|c|c|}
\hline \multirow{2}{*}{ Performance } & \multicolumn{3}{|c|}{$\begin{array}{c}\text { Activation energy at different } \\
\text { temperatures }\end{array}$} \\
\cline { 2 - 4 } & $\mathbf{7 0}^{\circ} \mathbf{C}$ & $\mathbf{7 0}^{\circ} \mathbf{C}$ & $\mathbf{6 0}^{\circ} \mathbf{C}$ \\
$\mathbf{6 0}^{\circ} \mathbf{C}$ & $\mathbf{5 0}^{\circ} \mathbf{C}$ & $\mathbf{5 0}^{\circ} \mathbf{C}$ \\
\hline$\varepsilon_{m}$ & 41.17 & 106.87 & 107.50 \\
\hline
\end{tabular}

By linear fitting to the formula (7), the value of the parameter $\mathrm{m}$ is obtained.

Accelerated aging test is carried out on the different stress levels of a solid propellant. The test is divided into four groups, each group has 5 tests, each test has 3 values, the ratio of the average value and the initial value is used as the test results, and the data obtained is in table 2 . 
Tab 2. Relationship between the aging life and the ratios of the test and initial mechanical property values of a solid propellant at different humidity-temperature combinations

\begin{tabular}{|c|c|c|c|c|c|c|c|}
\hline \multicolumn{2}{|c|}{$7^{\circ} \mathrm{C}, 70 \% \mathrm{RH}$} & \multicolumn{2}{|c|}{$70^{\circ} \mathrm{C}, 90 \% \mathrm{RH}$} & \multicolumn{2}{|c|}{$60^{\circ} \mathrm{C}, 70 \% \mathrm{RH}$} & \multicolumn{2}{|c|}{$60^{\circ} \mathrm{C}, 90 \% \mathrm{RH}$} \\
\hline d & $\mathbf{P} / \mathbf{P}_{0}$ & d & $\mathbf{P} / \mathbf{P}_{\mathbf{0}}$ & d & $\mathbf{P} / \mathbf{P}_{0}$ & d & $\mathbf{P} / \mathbf{P}_{\mathbf{0}}$ \\
\hline 2 & 0.8064 & 2 & 0.5383 & 4 & 0.8011 & 2.3 & 0.6678 \\
\hline 5.5 & 0.6706 & 3.5 & 0.4845 & 8 & 0.7036 & 4.3 & 0.5526 \\
\hline 9 & 0.6509 & 6 & 0.4629 & 12 & 0.6598 & 8.3 & 0.4678 \\
\hline 13 & 0.6176 & 10 & 0.2668 & 16 & 0.6555 & 11.3 & 0.3557 \\
\hline 16.6 & 0.5380 & 14 & 0.1626 & - & - & - & - \\
\hline
\end{tabular}

criterion. The relationship between the aging time and

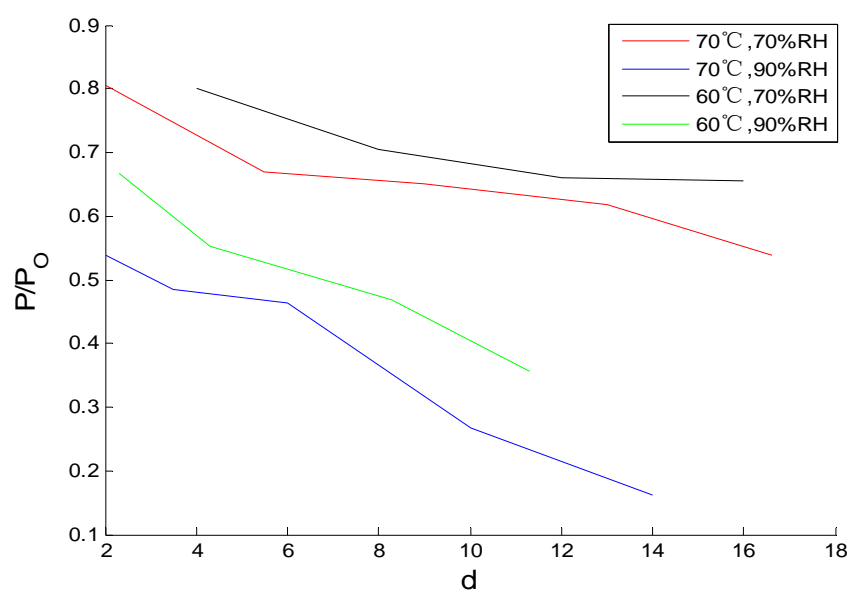

ratio of the mechanical properties with the original value is obtained by linear fitting. The four groups of data for the function relationship of aging life, temperature and humidity are obtained:

Tab 3.The function relationship of aging life, temperature and humidity

\begin{tabular}{|c|c|c|c|}
\hline NO. & $\begin{array}{c}\text { Aging } \\
\text { life(L) }\end{array}$ & Temperature(T) & Humidity(H) \\
\hline 1 & 19.0181 & 343.15 & 0.7 \\
\hline 2 & 3.4718 & 343.15 & 0.9 \\
\hline 3 & 29.0144 & 333.15 & 0.7 \\
\hline 4 & 6.8919 & 333.15 & 0.9 \\
\hline
\end{tabular}

Based on the four sets of data above, taking linear

Figure.1 Relationship between the aging life and the ratios of the test and initial mechanical property values of a solid propellant at different humidity-temperature combinations

From the figure above, it can be seen that with the time increasing, the mechanical properties of solid propellant drop seriously. And under the same humidity condition, when the temperature getting higher, the mechanical properties of the propellant decreases more rapidly, and the reaction rate increases with the increase of temperature. Under the same temperature condition, when the humidity getting higher, the mechanical properties of the propellant decreases more rapidly, too. Take both the temperature and humidity into consideration, which can be seen that the effect of humidity on the propellant aging life is more obviously than that of temperature. According to the test, the propellant will lose its effectiveness when exposed under $60 \%$ humidity condition for ten days, while that will cost much longer time for that in high temperature condition. The accuracy of life prediction can be improved by considering the factors of temperature and humidity working together.

Taking the mechanical properties of the propellant which is reduced to the original $50 \%$ as the failure fitting to the formula (10) and obtaining that :

$$
\begin{aligned}
& A=1.34 \times 10^{-5} \\
& m=-1.56 \\
& b=4.1510 \\
& c=5806.3
\end{aligned}
$$

The hydrothermal aging life model of the solid propellant is obtained as follow:

$$
L(H, T)=\frac{1.34 \times 10^{-5}}{H} T^{-1.56} e^{\frac{4.1510}{H}+\frac{5806.3}{T}}
$$

The life of a solid propellant at $25^{\circ} \mathrm{C}$ and $50 \%$ humidity condition is predicted by using the hydrothermal aging life model, and the predicted result is 11.7a.

\section{Conclusion}

(1) The hydrothermal aging life model based on the modified Arrhenius equation is deduced, and the model is used to predict the storage life of a solid propellant at temperature $25^{\circ} \mathrm{C}$ and humidity $50 \%$ condition, which result is $11.7 \mathrm{a}$;

(2) It can be seen from the experimental data that under 
the same humidity condition, when the temperature getting higher, the mechanical properties of the propellant decreases more rapidly, and the effect of humidity is the same as temperature which effect is more obviously. The accuracy of life prediction can be improved by considering the factors of temperature and humidity;

(3) Because of the limitation of experimental conditions, the model parameters can not be fitted well, and the accuracy of the model is affected.

\section{References}

[1] Hou L F. Composite Solid Propellant(Aerospace Press, Beijing, 1994)

[2] Zhao F, Chang X L. Hydrothermal Aging Life Model of A Composite Solid Propellant .J. Journal of Rocket Propulsion 34, 59 (2008)

[3] Zhang L, Chang X L, Lai J W. Estimation of HTPB Solid Propellant Life Span Based on Hydrothermal Accelerated Aging Experiment .J. Journal of Projectiles, Rockets, Missiles and Guidance 30, 148 (2010)

[4] Zhang $X$ J, Chang $X$ L, Chen S X et al. Hydrothermal Aging Testing and Life Assessment for Adhesive Interface of Solid Rocket Motor .J. Journal of Solid Rocket Technology 36, 27 (2013)

[5] Hou W H. A brief Introduction to the Establishment and Development of Chemical Kinetics .J. University Chemistry 22, 28 (2007)

[6] Gao D Y, He B, He S W et al. Discussion on Limitation of Arrhenius Methodology .J. Chinese Journal of Energetic Materials 14, 132 (2006)

[7] RELIASOFT COMPANY. Accelerated Life Testing Analysis Reference (ReliaSoft Publishing, 2001)

[8] Chen H J, Teng K N, Li B. A Research of Solid Rocket Motor Grain Storage Life Forecast Based on Modified Arrhenius Method .J. Journal of Projectiles, Rockets, Missiles and Guidance 31, 232 (2011)

[9] Zhang H, Luo H D, Du J. Linear Activation Energy Method for Predicting Service Life of Propellant .J. Journal of Solid Rocket Technology 25, 56 (2002)

[10]Luo H D, Zhang H, Du J. An Exploratory Study on Rapidly Predicting Service Life of Composite Solid Propellants .J. Journal of Solid Rocket Technology 23,31 (2000) 